\title{
Implementation of simulation-based crisis training in robotic thoracic surgery: how to improve safety and performance?
}

\author{
Jean-Marc Baste ${ }^{1,2}$, Benjamin Bottet ${ }^{1}$, Jean Selim ${ }^{3}$, Matthieu Sarsam ${ }^{1}$, Antoine Lefevre-Scelles ${ }^{2,3}$, \\ Marie-Melody Dusseaux ${ }^{3}$, Sébastien Franchina ${ }^{3}$, Anne-Sophie Palenzuela ${ }^{1}$, Abdeslam Chagraoui ${ }^{4}$, \\ Christophe Peillon ${ }^{1}$, Alexandre Thouroude ${ }^{5}$, Jean-Pierre Henry ${ }^{3}$, Jean-Michel M. Coq ${ }^{2,6}$, Louis Sibert ${ }^{2}$, \\ Cédric Damm ${ }^{3}$
}

\begin{abstract}
${ }^{1}$ Department of General and Thoracic Surgery, UNIROUEN, Inserm U1096, Rouen University Hospital, Normandie University, Rouen, France; ${ }^{2}$ MTC (Medical Training Center) Rouen, Normandie, France; ${ }^{3}$ Department of Anesthesia and Critical Care Medicine, UNIROUEN, Rouen University Hospital, Normandie University, Rouen, France; ${ }^{4}$ INSERM, U1239, Department of Medical Biochemistry, Rouen University Hospital, Normandie University, Rouen, France; ${ }^{5}$ Stan Institute, Nancy, France; ${ }^{6}$ Psychology Department, UFR Human and Social Sciences, EA 7475, Normandie University, Rouen, France

Correspondence to: Jean-Marc Baste, MD, PhD. Department of General and Thoracic Surgery, UNIROUEN, Inserm U1096, Rouen University Hospital, Normandie University, Rouen, France. Email: jean-marc.baste@chu-rouen.fr.
\end{abstract}

Submitted Apr 30, 2020. Accepted for publication Jul 14, 2020.

doi: $10.21037 /$ jtd-2020-epts-03

View this article at: http://dx.doi.org/10.21037/jtd-2020-epts-03

\section{Introduction}

Robotic-assisted thoracic surgery (RATS) is a new platform to perform minimally invasive surgery. This innovative tool has been developed to overcome surgical difficulties in video-assisted thoracic surgery (VATS) for major lung resection and advanced cases. Advantages as $3 \mathrm{D}$ vision, wrist instruments, ergonomics and precision are well known (Dylewski et al.) (1). However RATS, as VATS, presents some operative risks with rare but catastrophic adverse events, including vascular tear leading to potential uncontrolled hemorrhage $(2,3)$.

Because the operative surgeon works on a console located outside the operating field, RATS has radically modified teamwork and inter-professional communication (4). This may create a specific safety risk, which should be given special consideration. Cerfolio et al. (3) described the management of vascular complications during a robotic procedure using the 4P concept (Poise, Pressure, Prepare, Proximal control). However, this concept may be difficult to apply in a crisis situation such as a major vascular tear, not only for the console surgeon but also for the entire team. The stress level experienced by the surgeon is even higher than in VATS. Training in this process has become essential and should be addressed.

The use of checklists and simulation-based training have been demonstrated to improve the management of major crisis in the operating room and to decrease perioperative mortality $(5,6)$. Models of training have evolved from lifelike manikins to an entire range of systems, from synthetic models to highfidelity simulation suites. These models can be adapted to new technologies and used to train an entire surgical team.

To improve safety in RATS we have to follow a complete process with checklists and crisis checklists and also acquire a perfect knowledge of human factors $(5,6)$. To set this "safety and performance package" we have to learn and train with the entire team (7-9). Team simulation and crisis resource management (CRM) are innovative pedagogical tools but also represent a valuable evaluation method (8-11). Simulation has been described to enhance the skills of surgical teams (12).

Repetitive training sessions using high-fidelity simulation models have been advocated as a promising method for building teamwork skills because they mimic low-frequency, high-risk events in a safe, learner-friendly but stressful environment (13). Recently, training sessions using a scenario of airway obstruction were demonstrated to be effective in differentiating non-technical skills (NOTECHS) between thoracic surgery learners and experts (14).

In our center, we have developed a pilot simulation program in major lung resection to manage situations of 

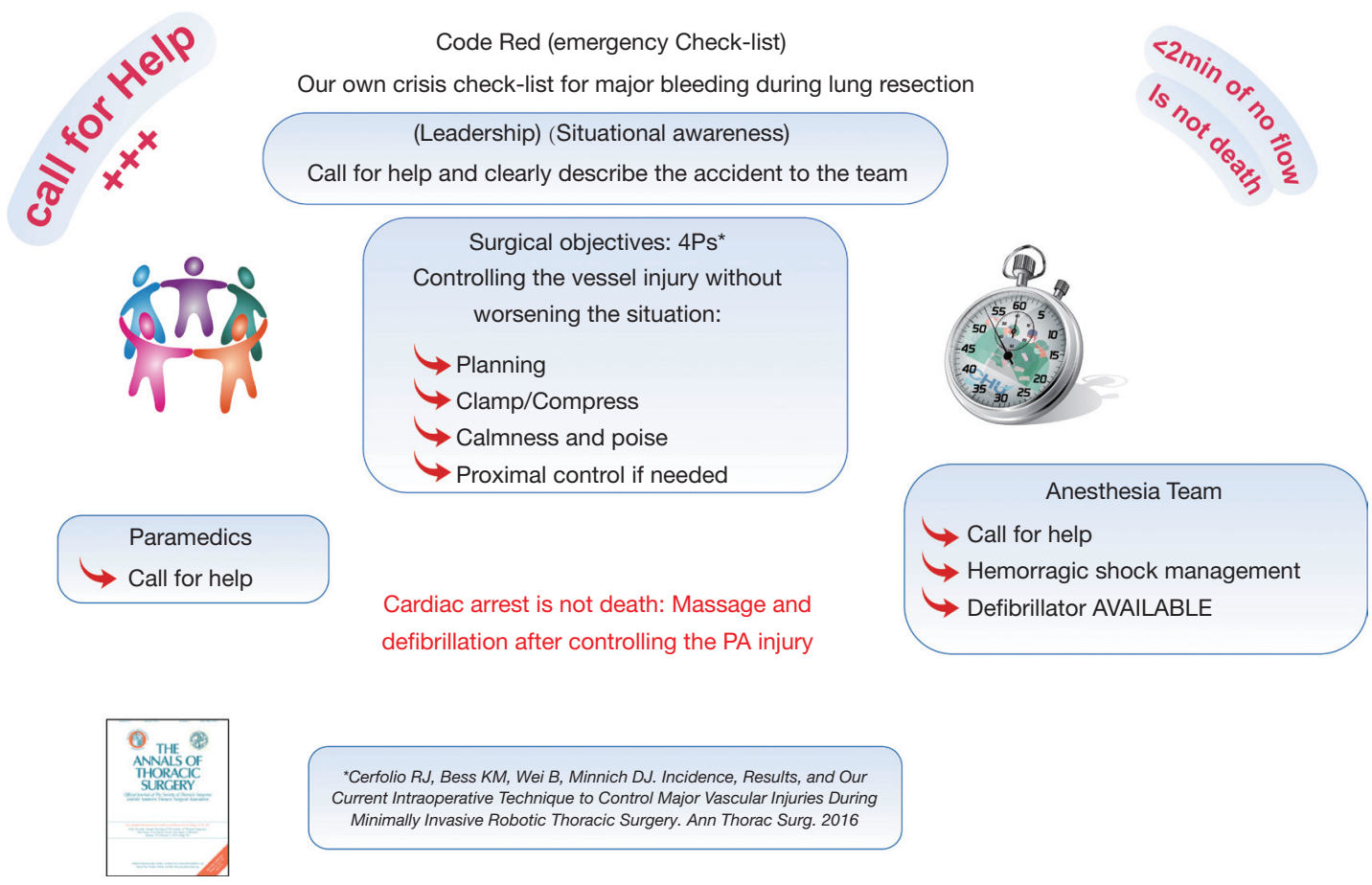

*Cerfolio RJ, Bess KM, Wei B, Minnich DJ. Incidence, Results, and Our Current Intraoperative Technique to Control Major Vascular Injuries During Minimally Invasive Robotic Thoracic Surgery. Ann Thorac Surg. 2016

Figure 1 Emergency checklist: "Code Red". CRM, crisis resource management; RATS, robotic-assisted thoracic surgery.

crisis and stress. This program began in 2014 with VATS and switched to RATS in 2015. The objective was to train the entire multidisciplinary and interprofessional perioperative team in the management of life-threatening crisis in the operating room. The aim of this paper is to describe the implementation of this program adapted to new technologies and using high-fidelity crisis models. Based on the experience gained, we show the feasibility of implementing simulationbased training in robotic thoracic surgery; we suggest improvements which integrate human factors knowledge to enhance team performance using training checklists and focusing on NOTECHS and stress coping strategies.

\section{Methods}

We have followed the guidelines and best practices of simulation (15) and have integrated all the different concepts to improve team effectiveness (training, checklists, human factors awareness, organizational design, stress awareness), presented in a recent review (16).

\section{Preparation}

Expert trainers attended "train-the-trainer" courses to improve the quality of the simulation program. These trainers were surgeons, anesthesiologists, nurse managers, human factors consultants and psychologists.

One of us (JMB) wrote the scenarios and built the program. Scenarios, objectives (technical skills, NOTECHS, stress management) and safety processes used for simulation were written after surgical morbidity and mortality conferences and root cause analysis (RCA), in accordance with simulation guidelines (post-hoc simulation of adverse events) (17).

Checklists adapted to RATS and emergency checklists adapted to major bleeding were used (Code Red, Figure 1). Non-technical aspects (leadership, decision-making, situational awareness, communication, cooperation, stress, and fatigue management) were a major focus of this program, because of their well-known impact on technical aspects $(18,19)$.

Information was given in lectures by the expert surgeon, anesthesiologist, psychologist, and human factors consultant to emphasize the role of cognitive aids (i.e., checklists) and to highlight the importance of NOTECHS in surgery and stress awareness $(18,19)$. In addition to making checklists accessible, all operating room staff that might be part of the team during a crisis were familiarized with their use. 

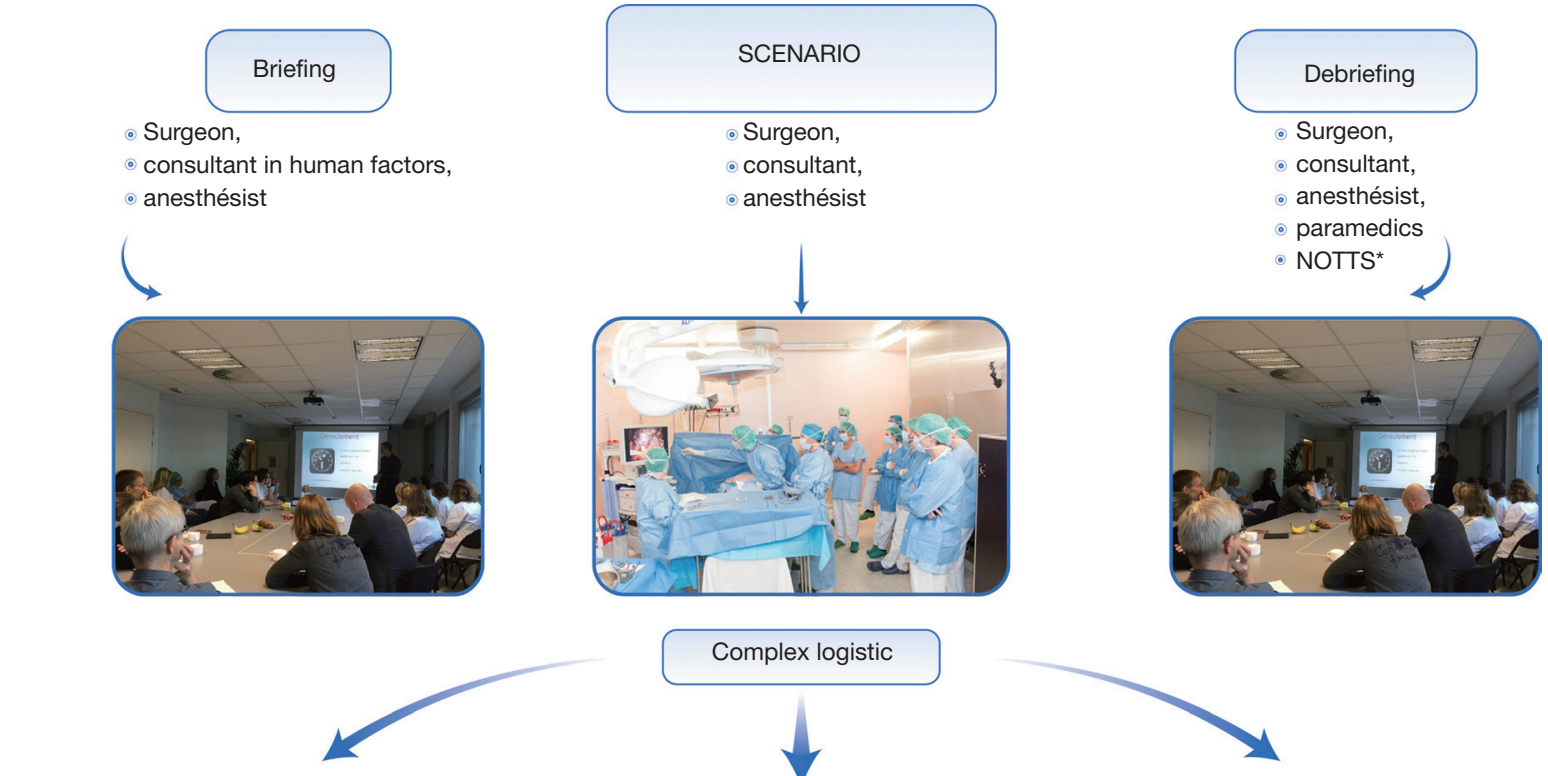

NOTTS*

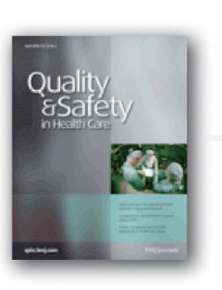

Technologies

Play scenario

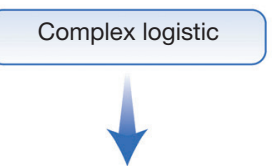

Video-conference

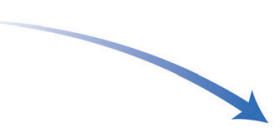

Figure 2 Organization of team simulation: briefing, scenario, debriefing.

\section{Logistics}

The logistics used were high-fidelity patient simulator (Manikin SimMan3G, Leardal ${ }^{\odot}$ ), operating room, robotic platform, camera for webcasting, technician for broadcasting, and a briefing and debriefing room (Figure 2).

\section{Clinical scenarios}

A hybrid model was most often used to reach a medium to high-fidelity model of controlled-uncontrolled bleeding during minimally invasive thoracic surgery (VATS and RATS) (Figure 3). Simulation was documented with actual scenarios of bleeding recorded in our VATSRATS operating room and more recently in the robotic suite. Different videos of controlled or uncontrolled bleeding during major lung resections were broadcast on the operating room screen to increase the fidelity of the scenarios and to raise the level of stress (Videos 1,2).

Another extremely stressful scenario was occasionally used: contralateral compressive pneumothorax with acute oxygen desaturation.

\section{Participants}

All members of an academic surgical team were invited to participate: surgeons, surgery fellows, surgery residents, medical students, anesthesiologists, anesthesiology fellows, anesthesiology residents, operating room nurses, nurse anesthetists, and operating room technicians; this was a multidisciplinary and interprofessional team (Figure 2). All these team members were recruited on a voluntary basis. Trained and novice staff were always pooled so we could use simulation as a training exercise as well as for evaluation. Sessions were always planned at the end of the 6-month rotation of residents (April and October).

\section{Simulation (Figure 3)}

In this simulation-based exercise, the facilitator (a senior 


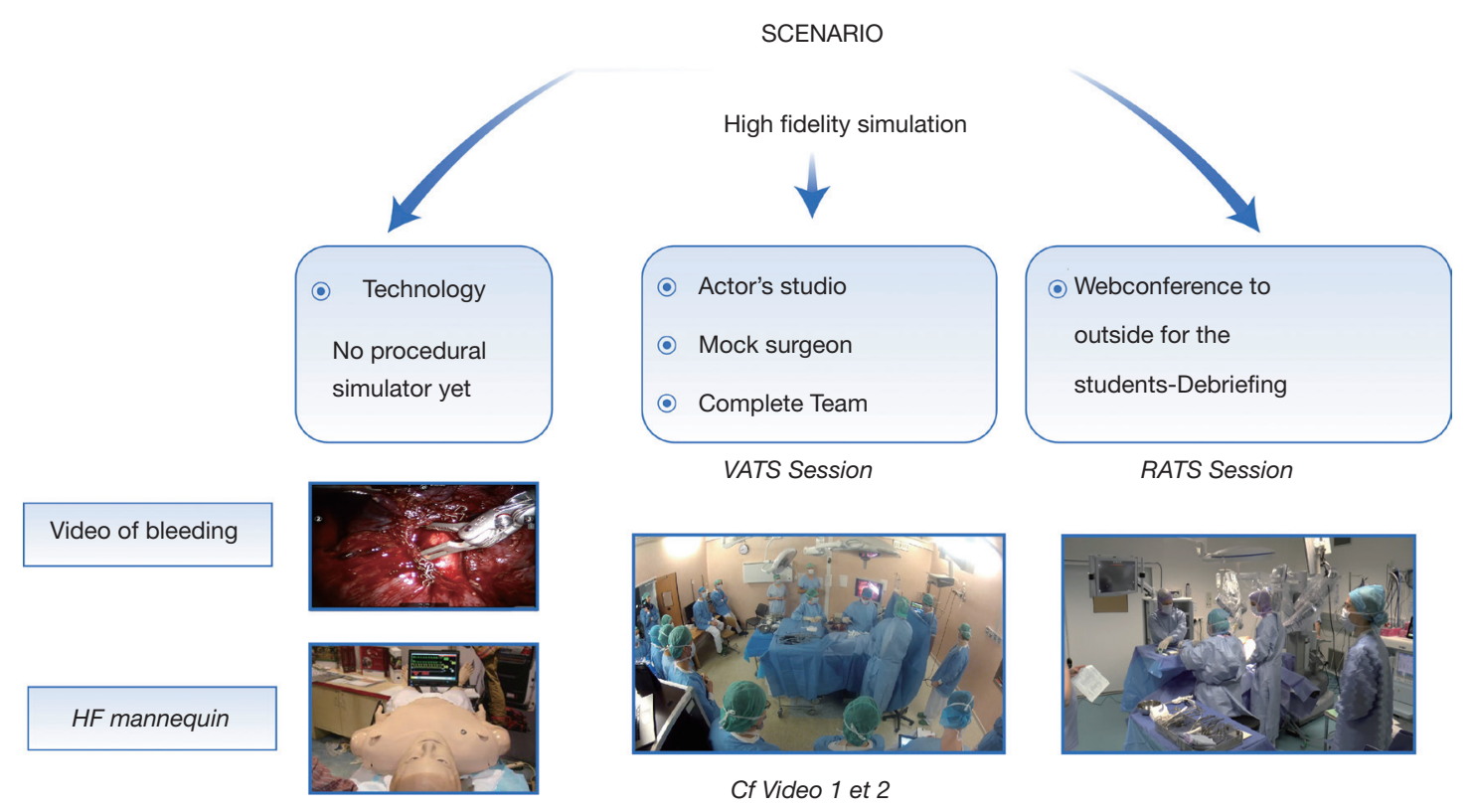

Figure 3 Scenario: operating room, high-fidelity model, facilitator, surgeon, assistant, nurses, robotic platform, camera for webcasting. VATS, video-assisted thoracic surgery; RATS, robotic-assisted thoracic surgery.

surgeon of the team) initiated the scenario and called for the sudden occurrence of a catastrophic and unexpected vessel tear. The senior surgeon could interrupt the teamwork to increase the stress level within the team. The console surgeon performing simulation (i.e., playing the main character) had to describe her/his plan and launch Code Red. During the simulated crisis, she/he had to focus on human factors and to describe the operative field, ensuring good situational awareness, good communication, good teamwork and stress management.

The anesthesiology team was tested for its ability to use recommended protocols in the framework of uncontrolled bleeding, such as the management of hemorrhagic shock with ventricular fibrillation and cardiac arrest. The anesthesiology team was also evaluated on its ability to maintain good communication and cooperation with the surgical team.

\section{Debriefing}

Each simulation session was followed by a debriefing session consisting in an individual and global analysis based on the self-assessment of each participant and on the comments made by independent observers, as proposed by Li et al. (20). Debriefing was conducted according to 3 phases-description, analysis, and application—as proposed by Jaye et al. (21). Two major questions were added at the end of each debrief: 'Did you gain new knowledge, skills or insights today?' and 'how confident do you feel that you will be able to apply what you learnt today in the future?'

Special consideration, highlighted by videos, was given to human factors within the surgical team: leadership, situational awareness, decision-making, cooperation, communication, stress management, and use of cognitive aids (pre-op and emergency checklists).

\section{Evaluation}

The simulation was evaluated in the short and long term. The short-term evaluation was done during the debriefing session; the long-term evaluation was done using the techniques of aviation security by an independent audit, which specifically evaluated human factors (Stan Institute, Nancy, France). The objectives of audits were to check our safety process (planning, checklist, human factors) and effectiveness (number of cases done, quality of life at work). The video was used to emphasize certain behaviors (cf. Audit French comment; global organization of a session in 2015).

\section{Results}

A video showing simulation sessions is accessible on CTS net platform (22). 
Table 1 Implementation over the time of our simulation training (2 sessions per year)

\begin{tabular}{|c|c|c|c|c|c|c|c|}
\hline Year & VATS CRMis & RATS CRMis & $\begin{array}{c}\text { RATS } \\
\text { CRMmtc }\end{array}$ & $\begin{array}{l}\text { Teams trained per } \\
\text { sessions }\end{array}$ & Scenarios & Simulation debriefing & Clinical audit \\
\hline 2014 & $x$ & & & 1 & U.B & Not structured & \\
\hline 2015 & & $\mathrm{X}$ & & 2 & U.B/C.P & Structured & $x$ \\
\hline 2016 & & $\mathrm{X}$ & & 2 & U.B & $\begin{array}{l}\text { Structured with } \\
\text { NOTECHS evaluation }\end{array}$ & \\
\hline 2017 & & $x$ & & 1 & U.B & NOTECHS & \\
\hline 2018 & & $x$ & & 1 & U.B & NOTECHS & \\
\hline 2019 & & & $x$ & & C.B & NOTECHS & $x$ \\
\hline
\end{tabular}

$\mathrm{X}$, done; RATS, robotic-assisted thoracic surgery; CRMis, in situ crisis resource management; CRMmts, crisis resource management in our Medical Training Center with a robotic platform Si (Intuitive); U.B, uncontrolled bleeding; C.B, controlled bleeding; C.P, compressive pneumothorax; Debriefing, Global Team effectiveness, Checklists audit, NOTECHS evaluations with the NOTECHS scale; Clinical audit, external audit by human factors consultants focused on NOTECHS and team performance.

\section{Organization (Table 1)}

First period: 2014-2016 (Video 3)

During this period, we built and designed the program and improved the scenarios and logistical issues. Sound broadcasting was the most difficult point to solve. It is very important to have a good quality of sound to evaluate the scenarios and NOTECHS, which is essential for good debriefing. Two sessions were necessary to achieve a better organization of simulation-based exercises and betterstructured debriefing. The implementation of this program prompted our center to provide logistical support.

\section{Second period: 2016-2019 (Video 4)}

Simulation sessions were organized every 6 months based on scenarios of controlled and uncontrolled bleeding during a robotic procedure (Videos 1,2). The bleeding scenario of the last simulation procedure was modified, with initial control of bleeding followed by a second tear during repair (Video 5). This is a classic event in thoracic surgery: a technical problem to repair a complex pulmonary tear and the risk of worsening the pulmonary artery tear.

\section{Crisis level of fidelity}

Based on RCA, the various technical and non-technical weaknesses during uncontrolled bleeding were recorded, analyzed and debriefed. This in situ hybrid simulation involving the entire surgical team, based on technical objectives using a high fidelity manikin and non-technical objectives, was considered effective by all participants to develop a high level of stress and to apply stress coping strategies. During all debriefings, participants described their stress and confirmed that coping with stress requires the development of a 2-level specific strategy. The first level was based on cognitive aids such as the use of emergency checklists (Code Red) involving supportive friends (surgeon, anesthesiologist and nurse). The second level was based on good teamwork with positive and clear communication, good timing of decision-making with clear description and explanation of the situation (situational awareness, anticipation). Critical points such as shouting, paradoxical injunction, bad decision-making, bad situational awareness, and the fast spread of stress to other members of the team were highlighted.

\section{Results of audits (Table 1)}

Auditing of each session by an independent audit company using the techniques of aviation security was an original initiative, which was beneficial in terms of identifying the problems and improvements to be made. During the period 2014-2019, audits identified clear improvements especially in non-technical fields. Calm, poise, and perfect adherence to safety processes were achieved even when dealing with major crises such as uncontrolled bleeding leading to cardiac arrest. In addition, successive audits noted the team's commitment to the entire program (briefing, simulation, debriefing), the consistent use of checklists, and adherence to the principle of robotic surgery and emergency conversion protocol. 


\section{Discussion}

The use of safety checklists in surgery has been demonstrated to reduce perioperative morbidity and mortality $(5,6)$. In simulation-based trials these checklists have been shown to improve the management of major crisis in the operating room $(6,15)$. These concepts are intended for the entire team working in the operating room. The question now is the implementation at hospital level. Ultimately, the question also arises of introduction in continuing education and accreditation programs (8). Hence, team training and CRM will likely become mandatory in the modern surgical curriculum even though its spread worldwide will take many years (8).

The concept of debriefing should be extended to the real surgical arena with improvement in team effectiveness and patient safety (19). Currently, this debriefing time is less developed than the time-out despite its integration in the WHO checklist. Debriefing allows for the identification of failures, near miss events, and successes, and provides an experiential learning modality. Debriefing has been shown to aid in reducing communication difficulties, which have been deemed the primary cause of human error in the surgical setting (23).

There is much literature on NOTECHS, team training and simulation with different programs (in situ simulation, training centers, different scenarios, different evaluations, low-high fidelity models, etc.) but worldwide implementation is not achieved $(8,24)$. Many reasons may explain this situation. Despite the fact that publications have shown improvement in team effectiveness, increased awareness of NOTECHS in surgeons, the use of team simulation is complex due to logistic issues and poor awareness of teamwork evaluation. It takes time to understand the different components of human factors (leadership, communication, teamwork, situational awareness) and the different scales used (NOTECHS scale, SAQ, ORMAQ) (20). We need to have dedicated specialists to evaluate teamwork properly. The non-technical science is not yet widespread, and this could explain the lack of adherence to team training programs and CRM in surgery. Another important point is the complexity of launching such costly programs, which is often time consuming.

In our opinion, the feasibility of this program is dependent on the ability of the facilitator surgeon to understand perfectly the objectives, master the human factors, and then adapt her/his playrole to the multidisciplinary team. Experience will make the difference. The most important point is to start the program with an open mind. We all know that error is human. The process of learning together with good feedback is fundamental. The objective of this program is to raise awareness of human factors and their impact on a team, on patient safety and on team effectiveness.

Simulation is likely one of the most impactful means to train teams in the appropriate use of the manuals, particularly when combined with other lower-cost methods to increase exposure. Yet, we believe it is fairly clear that, for cognitive aids to have their intended effect, team-training efforts are necessary in conjunction with broad clinical implementation efforts so that the concept penetrates deeply into the culture. For emergency manuals to be effective tools, they must be easily usable and contain the key content that is at the greatest risk of being missed.

Our crisis risk management program uses simulation and attempts to reproduce a rare and life-threatening event such as uncontrolled bleeding in RATS. This event is a true crisis for a surgical team with potential dysfunction and stress within the team. The major risk is the death of the patient, which is "unacceptable" in modern thoracic surgery mainly involving early lung cancer.

The level of stress induced by simulation models on the operative team depends on the facilitator surgeon who must have experience of catastrophic events during VATS and RATS major lung resection and who simulates "the crisis". Similarly, the quality of debriefing must take into account the experience of the facilitator who must have a clear vision of the objectives and be aware of the classic errors during a surgical crisis, e.g., blaming the nurse, paradoxical injunctions. Stress is widespread and should be instructed using different supports. We believe that our model could be assimilated with Stress Exposure Training (25-28).

Our hybrid scenario was a blend of mainly NOTECHS for the entire team and technical skills for the anesthesiologist (high-fidelity manikin with the management of hemorrhagic shock). The realism of the scenario depends on the console surgeon who must describe and explain the different steps of the crisis in real time. The team should adapt to the situation by adhering to the process (emergency checklist) and focusing on human factors: good situational awareness, good decision-making with clear, objective and safe communication with the entire team and with good cooperation. Our virtual scenario succeeded in increasing the level of stress and was representative of a real catastrophic event. This scenario could be improved by adding a technical task for the surgeon as this has been shown to have a positive effect on non-technical aspects (29). 


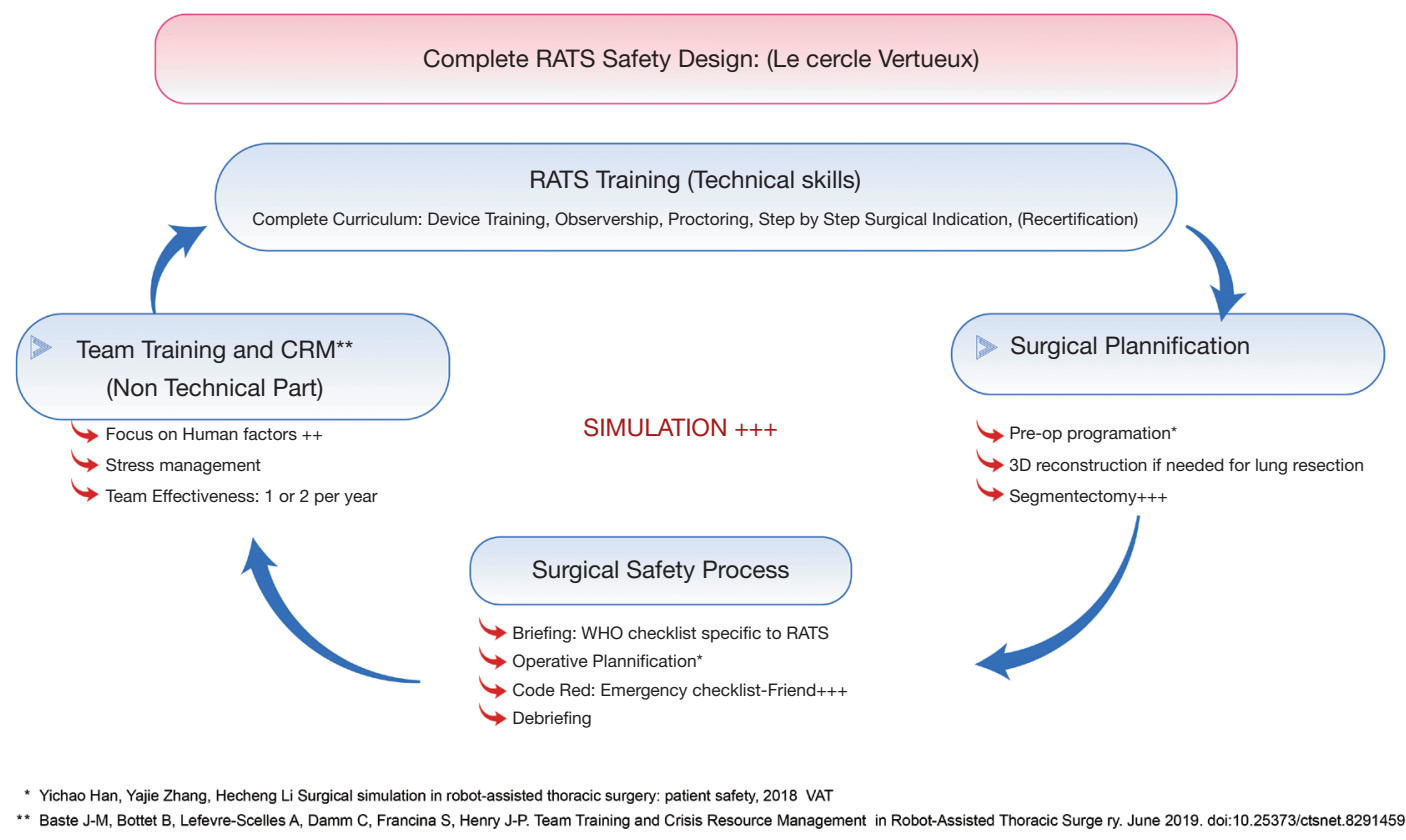

Figure 4 Our safety design: a comprehensive approach of robotic safety.

The main problem is the logistic issue and the cost of such a model. For our educational objectives we do not need to use a more complex model such as a perfused cadaver model (30).

Based on the simulation program and regular feedback, the safety process for robotic surgery was a complete process with cognitive aids and human factors enhancement (Figure 4). Team training and our simulation program have enabled us to establish an environment of confidence in our team. Attitudes have changed and regular feedback is considered beneficial to improve the quality and safety of our work. Confidence is difficult to measure but is the corner stone of effective teamwork. Stress can be measured by monitoring heart rate (26). This technique is interesting for research but not applicable in real life. Self-evaluation occurs progressively with experience and strategies to lower stress should be tested during simulation (27).

Crisis simulation also implies stress training. The primary purpose of stress exposure training is to prepare an individual to perform effectively in a stress environment. In broad terms, there are three overall goals of stress training: (I) gain knowledge and familiarity with the stress environment, (II) maintain effective performance under stress, and (III) build performance confidence (25-28). Simulation sessions provided enough stress to impact teamwork, especially for novice trainees such as nurses and young residents. Training is also an opportunity to highlight the mechanism of stress in a team and the fast spread to different team members. This emotive aspect is known to create a learning experience and enhance retention (25-28). This also enabled us to acknowledge our reaction to stress and to try to manage it properly by simple actions as breathing to calm down and refocusing on simple communication. Containing stress is clearly the "mission" of surgical team leaders (surgeons, anesthesiologists) who must train to cope with their own stress. This takes time but is a major issue because surgeons and anesthesiologists must be aware of their behavior (e.g., tachycardia, tremor, sweating, wavering) when facing a stressful situation. This simulation program helped us to focus on stress control.

In our opinion, the first line and the most important aspect in a crisis situation such as uncontrolled bleeding, is to share and find support; calling a friend or for assistance to support a surgeon under stress is the key message to the entire surgery team. That is why in our program when a crisis occurs it is mandatory to call a friend (6).

Our sessions were conducted every 6 months with good support from our team and novice colleagues (surgery residents, young operating room nurses, anesthesiology residents) regarding human factor concepts, the use of checklists and compliance with the entire safety process. Successive audits have shown that team attitudes have completely changed over time with full adherence to VATS 
and RATS checklists and protocols. During the study period we had one case of ventricular fibrillation at the beginning of a VATS case due to a probable cardiac event with 45 minutes of cardiac massage, ECMO support and successful resuscitation. This patient is still alive likely due to our safety process using the emergency checklist (Code Red) and very effective teamwork. This case report is currently under review and describes the importance of this program (31).

This simulation program could be easily exported to different centers. We have trained national and international teams for many years now. Some of our extrainees have reproduced this program in their center with good adherence from their local team, especially when they started their own robotic program (Caroline Rivera, MD, personal communication, French Society of Thoracic and Cardio-vascular, Rennes, France, 2019).

\section{Conclusions}

Simulation-based crisis training using models of catastrophic events in minimally invasive thoracic surgery should be part of all VATS and RATS programs to foster patient safety and team performance. The implementation of such a training program in our center received positive feedback from team members. This experience can be reproduced but requires the full commitment of medical and paramedical staff, and organizational and financial support from hospital management. We suggest that team training and CRM should be integrated into a lifelong learning program and could be used for certification in the future.

\section{Acknowledgments}

Prof. Baste and his team are grateful to Nikki SabourinGibbs and Laurent Thomas, MD, for editorial support. Funding: None.

\section{Footnote}

Provenance and Peer Review: This article was commissioned by the Guest Editors (Alessandro Brunelli and Enrico Ruffini) for the series "European Perspectives in Thoracic Surgery (2020) - the Seven Edition" published in fournal of Thoracic Disease. The article has undergone external peer review.

Conflicts of Interest: All authors have completed the ICMJE uniform disclosure form, available at: http://dx.doi. org/10.21037/jtd-2020-epts-03. The series "European Perspectives in Thoracic Surgery (2020) - the Seven Edition" was commissioned by the editorial office without any funding or sponsorship. JMB reports personal fees from Intuitive Surgery, personal fees from Johnson and Johnson, personal fees from Medtronic, during the conduct of the study. ALS reports other from Medtronic, outside the submitted work. CP reports personal fees from covidien, during the conduct of the study. The other authors have no other conflicts of interest to declare.

Ethical Statement: The authors are is accountable for all aspects of the work in ensuring that questions related to the accuracy or integrity of any part of the work are appropriately investigated and resolved.

Open Access Statement: This is an Open Access article distributed in accordance with the Creative Commons Attribution-NonCommercial-NoDerivs 4.0 International License (CC BY-NC-ND 4.0), which permits the noncommercial replication and distribution of the article with the strict proviso that no changes or edits are made and the original work is properly cited (including links to both the formal publication through the relevant DOI and the license). See: https://creativecommons.org/licenses/by-nc-nd/4.0/.

\section{References}

1. Dylewski MR, Lazzaro RS. Robotics - The answer to the Achilles' heel of VATS pulmonary resection. Chin J Cancer Res 2012;24:259-60.

2. Flores RM, Ihekweazu U, Dycoco J, et al. Video-assisted thoracoscopic surgery (VATS) lobectomy: catastrophic intraoperative complications. J Thorac Cardiovasc Surg 2011;142:1412-7.

3. Cerfolio RJ, Bess KM, Wei B, et al. Incidence, Results, and Our Current Intraoperative Technique to Control Major Vascular Injuries During Minimally Invasive Robotic Thoracic Surgery. Ann Thorac Surg 2016;102:394-9.

4. Catchpole K, Bisantz A, Hallbeck MS, et al. Human factors in robotic assisted surgery: Lessons from studies 'in the Wild'. Appl Ergon 2019;78:270-6.

5. Haynes AB, Weiser TG, Berry WR, et al. A surgical safety checklist to reduce morbidity and mortality in a global population. N Engl J Med 2009;360:491-9.

6. Arriaga AF, Bader AM, Wong JM, et al. Simulationbased trial of surgical-crisis checklists. N Engl J Med 2013;368:246-53. 
7. McEwan D, Ruissen GR, Eys MA, et al. The Effectiveness of Teamwork Training on Teamwork Behaviors and Team Performance: A Systematic Review and Meta-Analysis of Controlled Interventions. PLoS One 2017;12:e0169604.

8. WHO. Patient Safety Curriculum Guide - Multiprofessional Edition. 2011.

9. Howell AM, Panesar SS, Burns EM, et al. Reducing the burden of surgical harm: a systematic review of the interventions used to reduce adverse events in surgery. Ann Surg 2014;259:630-41.

10. Undre S, Koutantji M, Sevdalis N, et al. Multidisciplinary crisis simulations: the way forward for training surgical teams. World J Surg 2007;31:1843-53.

11. Okuda Y, Bryson EO, DeMaria S Jr, et al. The utility of simulation in medical education: what is the evidence? $\mathrm{Mt}$ Sinai J Med 2009;76:330-43.

12. Aggarwal R, Undre S, Moorthy K, et al. The simulated operating theatre: comprehensive training for surgical teams. Qual Saf Health Care 2004;13 Suppl 1:i27-32.

13. Weis JJ, Croft CL, Bhoja R, et al. Multidisciplinary Simulation Activity Effectively Prepares Residents for Participation in Patient Safety Activities. J Surg Educ 2019;76:e232-7.

14. Bierer J, Memu E, Leeper WR, et al. Development of an In Situ Thoracic Surgery Crisis Simulation Focused on Nontechnical Skill Training. Ann Thorac Surg 2018;106:287-92.

15. Aggarwal R, Mytton OT, Derbrew M, et al. Training and simulation for patient safety. Qual Saf Health Care 2010;19 Suppl 2:i34-43.

16. Buljac-Samardzic M, Doekhie KD, van Wijngaarden JDH. Interventions to improve team effectiveness within health care: a systematic review of the past decade. Hum Resour Health 2020;18:2.

17. Johna S, Tang T, Saidy M. Patient safety in surgical residency: root cause analysis and the surgical morbidity and mortality conference--case series from clinical practice. Perm J 2012;16:67-9.

18. Hull L, Arora S, Aggarwal R, et al. The impact of nontechnical skills on technical performance in surgery: a systematic review. J Am Coll Surg 2012;214:214-30.

19. Yule S, Flin R, Paterson-Brown S, et al. Non-technical skills for surgeons in the operating room: A review of the literature. Surgery 2006;139:140-9.

20. Li N, Marshall D, Sykes M. Systematic review of methods for quantifying teamwork in the operating theatre. BJS Open 2018;2:42-51.

21. Jaye P, Thomas L, Reedy G. 'The Diamond': a structure for simulation debrief. Clin Teach 2015;12:171-5.

22. Baste JM, Bottet B, Lefevre-Scelles A. Team Training and Crisis Resource Management in Robot-Assisted Thoracic Surgery. 2019. Available online: https://ctsnet. figshare.com/articles/Team_Training_and_Crisis_ Resource_Management_in_Robot-Assisted_Thoracic_ Surgery/8291459/1

23. Zuckerman SL, France DJ, Green C, et al. Surgical debriefing: A reliable roadmap to completing the patient safety cycle. Neurosurg Focus 2012;33:E4.

24. Selvy M, Le Roy B, Abjean A, et al. The increasing prominence of "non-surgical" articles in surgical literature. J Visc Surg 2020;157:37-41.

25. Wetzel CM, George A, Hanna GB, et al. Stress management training for surgeons-a randomized, controlled, intervention study. Ann Surg 2011;253:488-94.

26. Grantcharov PD, Boillat T, Elkabany S, et al. Acute mental stress and surgical performance. BJS Open 2018;3:119-25.

27. Wetzel CM, Black SA, Hanna GB, et al. The effects of stress and coping on surgical performance during simulations. Ann Surg 2010;251:171-6.

28. Driskell SE, Salas E. Stress Exposure Training. Overcoming the effects of stress on military performance: Human factors, training, and selection strategies. In: Gal R, Mangels-dorff D. editors. Handbook of Military Psychology. Oxford: John Wiley \& Sons, 1991:183-93.

29. Sparks JL, Crouch DL, Sobba K, et al. Association of a Surgical Task During Training with Team Skill Acquisition Among Surgical Residents: The Missing Piece in Multidisciplinary Team Training. JAMA Surg 2017;152:818-25.

30. Delpech PO, Danion J, Oriot D, et al. SimLife a new model of simulation using a pulsated revascularized and reventilated cadaver for surgical education. J Visc Surg 2017;154:15-20.

31. Rinieri P, Selim J, Le Guillou V, et al. Crisis checklist (Code Red) for the management of cardiac arrest during minimally invasive thoracic surgery: case report. J Cardiothorac Surg 2020;15:173.

Cite this article as: Baste JM, Bottet B, Selim J, Sarsam M, Lefevre-Scelles A, Dusseaux MM, Franchina S, Palenzuela AS, Chagraoui A, Peillon C, Thouroude A, Henry JP, Coq JMM, Sibert L, Damm C. Implementation of simulation-based crisis training in robotic thoracic surgery: how to improve safety and performance? J Thorac Dis 2021;13(Suppl 1):S26-S34. doi: 10.21037/jtd-2020-epts-03 